Sir,

\section{Patients attending eye clinic have poor left right discrimination}

Problems with left right discrimination can cause medical errors including investigation or treatment of the wrong eye, which may result in potentially serious consequences. ${ }^{1}$ The ability of doctors and medical students to correctly discriminate left and right has already been extensively explored ${ }^{2}$ and because of varying ability to discriminate effectively, numerous safety checks exist to prevent errors. Previous studies have also demonstrated differences in ability to discriminate based on educational level ${ }^{3}$ and sex. ${ }^{4}$ It has not been previously explored whether language preference has any bearing on left right discrimination. It seemed that patients that attended our clinics who could correctly lateralise were less frequent than the quoted rates of between 80.5 and $73.4 \% .{ }^{4,5}$ We decided to look at the ability of patients attending our outpatient department to discriminate left and right and look for differences between sexes and language used in the consultation, Welsh or English.

Patients attending outpatient ophthalmic clinics in South West Wales were asked to 'look to their right' as part of the routine slit-lamp examination and tallies of correct responses were taken, along with details of sex and language of consultation. The language used was based on that of patient preference. A positive response was defined as the patient either immediately looking to the right, as requested, or looking to the right after some period of hesitation. Of a total of 2309 patients seen, $1417(61.4 \%)$ correctly looked to the right when asked. There was no significant difference $(P=0.5552)$ between the sexes in correct left right discrimination, however, 285 out of 390 Welsh-speaking patients looked to the right (73.1\%) vs 1132 out of 1919 (59\%) English-speaking patients, which was statistically significant $(P=0.0011)$.

We found that rates of correct left right discrimination among clinic attendees were significantly lower than previously published rates and that the potential for medical errors is thus higher than thought. Our observed variance in ability to lateralise may be due to, among other factors, hearing problems in our population with the more vocally distinct 'Dde' and 'Chwith', for right and left, respectively, in the Welsh language, being more easily understood.

\section{Conflict of interest}

The authors declare no conflict of interest.

\section{References}

1 Seiden SC, Barach P. Wrong-side/wrong-site, wrong procedure, and wrong patients adverse effects. Arch Surg 2006; 141: 931-939.

2 Gormley GJ, Dempster M, Best R. Right-left discrimination among medical students: questionnaire and psychometric study. BMJ 2008; 337: a2826.

3 Ofte SH. Right-left discrimination: effects of handedness and educational background. Scand J Psychol 2002; 43: 213-219.

4 Jordan K, Wustenberg T, Jaspers-Fever F, Fellbrich A, Peters M. Sex differences in left/right confusion. Cortex 2006; 42: 69-78.

5 Hannay HJ, Ciaccia PJ, Kerr JW, Barrett D. Self-report of right-left confusion in college men and women. Percept Mot Skills 1990; 70: 451-457.

GS Williams and DJ Eddyshaw

Department of Ophthalmology, Singleton Hospital Wales, UK

E-mail: gwynwilliams@doctors.org.uk

Eye (2011) 25, 1243; doi:10.1038/eye.2011.137; published online 17 June 2011

Sir,

Recent trends in the rate of trabeculectomy in England

We previously reported a sharp decline in the annual rate of trabeculectomy operations in England: from a peak of 38.7 episodes per 100000 population in 1995/6 towards a plateau around 11 episodes per 100000 in 2003/41 (where 2003/4 refers to the NHS financial year 1st April 2003 to 31st March 2004). As discussed previously, ${ }^{1}$ this was principally due to the introduction of new topical agents with improved control of intraocular pressure, and was accompanied by similar decreases in trabeculectomy rates throughout Britain, Europe, Canada, and the United States of America. Tatham and Sarodia ${ }^{2}$ recently described an increase of $149 \%$ in the annual number of trabeculectomy operations at Leicester Royal Infirmary, from 71 in 2005 to 177 in 2009, and suggested that the national decline in trabeculectomy may have begun to reverse.

We have used recent data to study trends in the number and rate of hospital episodes of trabeculectomy in England as a whole (Table 1 and Figure 1). Hospital

Table 1 Annual number of trabeculectomy operations, and annual rate of trabeculectomy operations per 100000 population, in England from 1989/1990 to 2009/2010

\begin{tabular}{|c|c|c|c|c|c|c|c|c|c|c|c|}
\hline Year & 1989 & 1990 & 1991 & 1992 & 1993 & 1994 & 1995 & 1996 & 1997 & 1998 & \\
\hline No. of trabeculectomy operations & 8682 & 10514 & 12768 & 15054 & 17065 & 17496 & 18676 & 17350 & 14244 & 11859 & \\
\hline $\begin{array}{l}\text { Rate of trabeculectomy } \\
\text { (episodes } / 100000 \text { population) }\end{array}$ & 18.3 & 22.0 & 26.7 & 31.4 & 35.5 & 36.3 & 38.7 & 35.9 & 29.4 & 24.4 & \\
\hline Year & 1999 & 2000 & 2001 & 2002 & 2003 & 2004 & 2005 & 2006 & 2007 & 2008 & 2009 \\
\hline No. of trabeculectomy operations & 8948 & 7407 & 6268 & 5663 & 5319 & 5289 & 5208 & 4978 & 5433 & 5281 & 5679 \\
\hline $\begin{array}{l}\text { Rate of trabeculectomy } \\
\text { (episodes } / 100000 \text { population) }\end{array}$ & 18.3 & 15.1 & 12.7 & 11.4 & 10.7 & 10.6 & 10.2 & 9.8 & 10.7 & 10.2 & 11.0 \\
\hline
\end{tabular}

